\title{
CREB Family Gene
}

National Cancer Institute

\section{Source}

National Cancer Institute. CREB Family Gene. NCI Thesaurus. Code C38534.

Ubiquitously or widely expressed human CREB Family Genes (bZIP/CREB Family) encode conserved cAMP Responsive Element Binding Proteins, nuclear bZIP domain dimeric transcription factors that bind to octameric DNA palindrome CAMP-response elements (CRE) present in many viral and cellular promoters and induce gene transcription in response to CAMP signaling pathways. CREB proteins bind to DNA as a homodimer or a heterodimer with JUN/c-Jun or ATF2/CREBP1. Increased CAMP levels following stimulation activate CAMP-dependent protein kinase A, which phosphorylates CREB proteins that stimulate transcription of CAMP-responsive genes. Calcium-regulated CREB transcription factors integ rate calcium and CAMP signals. CAMP pathways provide a chief means by which cellular growth, differentiation, and function can be influenced by extracellular signals. ( $\mathrm{NCl})$ 